\title{
On the containment problem
}

\author{
T. Szemberg ${ }^{1}$ - J. Szpond ${ }^{2}$
}

Received: 7 January 2016 / Accepted: 22 October 2016 / Published online: 28 October 2016

(C) The Author(s) 2016. This article is published with open access at Springerlink.com

\begin{abstract}
The purpose of this note is to provide an overview of the containment problem for symbolic and ordinary powers of homogeneous ideals, related conjectures and examples. We focus here on ideals with zero dimensional support. This is an area of ongoing active research. We conclude the note with a list of potential promising paths of further research.
\end{abstract}

Keywords Symbolic power $\cdot$ Fat points - Waldschmidt constants

Mathematics Subject Classification $14 \mathrm{C} 20 \cdot 14 \mathrm{~J} 26 \cdot 14 \mathrm{~N} 20 \cdot 13 \mathrm{~A} 15 \cdot 13 \mathrm{~F} 20$

\section{Introduction}

Let $I \subset R=\mathbb{K}\left[x_{0}, \ldots, x_{N}\right]$ be a homogeneous ideal. Such an ideal determines various sequences of associated ideals. The most natural one is that of ordinary powers of $I$ :

$$
I^{0}=R \supset I^{1}=I \supset I^{2} \supset I^{3} \supset I^{4} \supset \cdots
$$

If $I=\left\langle f_{1}, \ldots, f_{n}\right\rangle$ is given explicitly in terms of generators, then the $r$-th ordinary power of $I$ is generated by products

$$
f_{i_{1}} \cdots f_{i_{r}} \text { with } i_{1}, \ldots, i_{r} \in\{1, \ldots, n\}
$$

\section{T. Szemberg}

tomasz.szemberg@gmail.com

\section{J. Szpond}

szpond@up.krakow.pl

1 Polish Academy of Sciences, Institute of Mathematics, ul. Śniadeckich 8, 00-656 Warsaw, Poland

2 Department of Mathematics, Pedagogical University of Cracow, Podchorążych 2, 30-084 Kraków, Poland 
Another important sequence of ideals associated to $I$ is the sequence of symbolic powers of $I$ :

$$
I^{(0)}=R \supset I^{(1)}=I \supset I^{(2)} \supset I^{(3)} \supset I^{(4)} \supset \cdots .
$$

Recall that for $m \geq 0$ the $m$-th symbolic power of $I$ is defined as

$$
I^{(m)}=\bigcap_{P \in \operatorname{Ass}(I)}\left(I^{m} R_{P} \cap R\right),
$$

where $\operatorname{Ass}(I)$ is the set of associated primes of $I$.

Contrary to ordinary powers, even if $I$ is given explicitly by a set of generators $I=$ $\left\langle f_{1}, \ldots, f_{n}\right\rangle$, it is very difficult in general to determine generators of $I^{(m)}$ for $m \geq 2$. On the other hand, if $I$ is a radical ideal and $V=V(I)$ is the reduced subscheme in $\mathbb{P}^{N}$ defined by $I$, then the symbolic power $I^{(m)}$ consists of all polynomials in $R$ vanishing to order at least $m$ along $V$. This is a consequence of the famous Nagata-Zariski Theorem, see [16, Theorem 3.14] for prime ideals and [28, Corollary 2.9] for radical ideals. Thus, in this case, symbolic powers have clear geometric interpretation.

Research presented in this note is motivated by the following main question.

Problem 1.1 (The containment problem) Decide for which $m$ and $r$ there is the containment

$$
I^{(m)} \subset I^{r} .
$$

Remark 1.2 Note that for the reverse containment i.e.

$$
I^{r} \subset I^{(m)}
$$

the analogous question has a very simple answer. The containment holds if and only if $m \leq r$ [3, Lemma 8.4.1].

It came in a sense as a surprise that there does exist a uniform and simple stated answer to the Containment Problem. It was discovered by Ein, Lazarsfeld and Smith in characteristic zero, see [15] and by Hochster and Huneke in positive characteristic, see [20]. Both papers builded upon ground breaking ideas of Swanson in [29].

Theorem 1.3 (Ein-Lazarsfeld-Smith, Hochster-Huneke) Let $I \subset \mathbb{K}\left[x_{0}, \ldots, x_{N}\right]$ be a homogeneous ideal such that every component of its zero locus $V(I)$ has codimension at most e. Then the containment

$$
I^{(m)} \subset I^{r}
$$

holds for all $m \geq$ er.

The above Theorem is a powerful and elegant result. We focus here on ideals defining zerodimensional subschemes of $\mathbb{P}^{N}$. In this case the containment condition in the above Theorem is $m \geq N r$. It is natural to wonder to what extend the bound $m \geq N r$ is sharp. A striking fact is that there is not a single example known (at least to the authors of the present note), where for all $r$ one really needs $m \geq N r$ for the containment (2) to hold, i.e. the containment holds for lower values of $m$. This has prompted Huneke to ask the following question addressing the first non-trivial case of Theorem 1.3 i.e. that of points in $\mathbb{P}^{2}$.

Problem 1.4 (Huneke) Let $I$ be a saturated ideal of a reduced finite set of points in $\mathbb{P}^{2}$. Does then the containment

$$
I^{(3)} \subset I^{2}
$$

hold? 
Positive answer to Problem 1.4 obtained in an ample family of special cases and additional experimental data led to the following more general question, see [3, Conjecture 8.4.2], [6, Conjecture 1.1], [18, Conjecture 4.1.1], [4, Conjecture 3.2].

Problem 1.5 (Bocci, Harbourne, Huneke) Let $I$ be a saturated ideal of a finite set of reduced points in $\mathbb{P}^{N}$. Does the containment

$$
I^{(m)} \subset I^{r}
$$

hold for $m \geq N r-(N-1)$ ?

This note gives an overview of verified cases and constructed counterexamples which have been discovered recently. There is also a number of open questions in this area of active current interest and ongoing investigations, see e.g. [22,24,26] for recent contributions.

\section{Symbolic powers for ideals of points in projective spaces}

Since in this note we focus on ideals supported on sets of reduced points or, sometimes more generally, on sets of fat points, we recall here briefly the main notions. In the particular situation of points, symbolic powers can be defined in an easier and more accessible way than provided in (1). Let $Z=\left\{P_{1}, \ldots, P_{s}\right\}$ be a union of reduced points in $\mathbb{P}^{N}$. For a fixed $i \in\{1, \ldots, s\}$ let $I\left(P_{i}\right)$ denote the ideal of polynomials vanishing at $P_{i}$. Then obviously the ideal of the set $Z$ satisfies:

$$
I(Z)=I\left(P_{1}\right) \cap \cdots \cap I\left(P_{S}\right) .
$$

The symbolic powers of $I(Z)$ are given in the same way:

$$
I(Z)^{(m)}=I\left(P_{1}\right)^{m} \cap \cdots \cap I\left(P_{S}\right)^{m}
$$

for any $m \geq 1$. Note that any point $P \in \mathbb{P}^{N}$ is a complete intersection, hence $I(P)^{(m)}=$ $I(P)^{m}$ for all $m \geq 1$. Indeed since the powers of a complete intersection ideal are arithmetically Cohen-Macaulay the claim follows from the Unmixedness Theorem [16, Corollary 18.14]. So that the right hand side of (4) is the intersection of symbolic powers of ideals of each component of $Z$.

The same holds in a slightly more general setting.

Definition 2.1 (Fat points scheme) A subscheme $Z \subset \mathbb{P}^{N}$ is a fat points scheme if its ideal $I(Z)$ has the form

$$
I(Z)=I\left(P_{1}\right)^{m_{1}} \cap \cdots \cap I\left(P_{s}\right)^{m_{s}}
$$

for points $P_{1}, \ldots, P_{s}$ in the projective space $\mathbb{P}^{N}$ and positive integers $m_{1}, \ldots, m_{s}$.

In this situation $I(Z)$ is a called a fat points ideal.

For symbolic powers of a fat points ideal $I(Z)=I\left(P_{1}\right)^{m_{1}} \cap \cdots \cap I\left(P_{s}\right)^{m_{s}}$ we have

$$
I(Z)^{(m)}=I\left(P_{1}\right)^{m m_{1}} \cap \cdots \cap I\left(P_{s}\right)^{m m_{s}} .
$$




\section{General statements motivated by Problem 1.5}

To begin with note that the constant $N$ appearing in the statement of Theorem 1.3 can not be lowered. A series of examples was constructed by Bocci and Harbourne in [5]. The main idea to study the so called star configurations of points in $\mathbb{P}^{N}$ goes back to Ein.

Definition 3.1 (Star configuration of points) We say that $Z \subset \mathbb{P}^{N}$ is a star configuration of degree $d$ (or a $d$-star for short) if $Z$ consists of all intersection points of $d \geq N$ general hyperplanes in $\mathbb{P}^{N}$. By intersection points we mean the points which belong to exactly $N$ of given $d$ hyperplanes.

The assumption general in the Definition means that any $N$ of $d$ given hyperplanes meet in a single point and there is no point belonging to $N+1$ or more hyperplanes. Star configurations can be defined much more generally. They form an interesting and combinatorially easy to describe class of examples occurring in various situations in algebra and geometry. We refer to [17] for a very nice introduction to this circle of ideas.

Now the result of Bocci and Harbourne is as follows, see [5, Theorem 2.4.3].

Proposition 3.2 Let $Z \subset \mathbb{P}^{N}$ be a d-star. Let I be the ideal of $Z$. Then for any $c<\frac{d-N+1}{d} N$ there exist $m$ and $r$ such that

$$
\frac{m}{r} \leq c \text { and } I^{(m)} \nsubseteq I^{r} .
$$

Since $d$ can be taken arbitrarily large, we see that there is no constant lower than $N$ which would satisfy the statement of Theorem 1.3. Investigating into this problem has prompted Bocci and Harbourne to introduce in [5] a new interesting invariant, measuring in a sense a discrepancy between symbolic and ordinary powers.

Definition 3.3 (Resurgence) Let $I$ be a homogeneous ideal. The resurgence of $I$ is the real number

$$
\rho(I)=\sup \left\{\frac{m}{r}: I^{(m)} \nsubseteq I^{r}\right\} .
$$

This is a delicate invariant and has been computed only in few special cases. One of them is

$$
\rho(I)=\frac{d-N+1}{d}
$$

for a $d$-star in $\mathbb{P}^{N}$, see [5, Theorem 2.4.3]. The importance of $\rho(I)$ follows from the fact that there is the containment

$$
I^{(m)} \subset I^{r} \text { provided } \frac{m}{r}>\rho(I) .
$$

Of course the applicability of (5) is limited by the computability of $\rho(I)$. There are however results along these lines which in certain situations lead to interesting consequences. Before stating sample results of this kind we need to introduce further invariants.

Definition 3.4 (The initial degree) Let $I=\oplus_{d \geq 0} I_{d}$ be a homogeneous ideal in $R$. Then the initial degree $\alpha(I)$ is defined as the least number $d$ such that $I_{d} \neq 0$. In other words, $\alpha(I)$ is the least degree of a non-zero polynomial in $I$.

Alternatively, denoting by $M=\left\langle x_{0}, \ldots, x_{N}\right\rangle$ the irrelevant ideal of $R$, the initial degree $\alpha(I)$ may thought of as the $M$-adic order of $I$, i.e. the largest $t$ such that $I \subset M^{t}$.

The next invariant, the Castelnuovo-Mumford regularity reg $(I)$ of $I$ provides a measure, in terms of the minimal free resolution, of how complicated the ideal $I$ is. 
Definition 3.5 (Castelnuovo-Mumford regularity) Let $I \subset R$ be a homogeneous ideal and let

$$
0 \longrightarrow \cdots \longrightarrow F_{j} \longrightarrow \cdots \longrightarrow F_{0} \longrightarrow I \longrightarrow 0
$$

be the minimal free resolution of $I$ over $R$. Let $f_{j}$ be the maximal degree of a generator in a minimal set of generators of $F_{j}$. Then

$$
\operatorname{reg}(I)=\max \left\{f_{j}-j, j \geq 0\right\} .
$$

The following useful result is Lemma 2.3.4 in [5] and Lemma 2.1 in [4].

Proposition 3.6 (Postulation Containment Criterion) Let I be a homogeneous (not necessarily saturated) ideal defining a 0 -dimensional subscheme in $\mathbb{P}^{N}$. If the inequality

$$
r \cdot \operatorname{reg}(I) \leq \alpha\left(I^{(m)}\right)
$$

holds, then there is the containment

$$
I^{(m)} \subset I^{r} .
$$

Remark 3.7 (Computability of $\alpha(I)$ ) Note that the initial degree of a symbolic power of an ideal is not easy to compute in general. Suffices it to say that the famous Nagata Conjecture predicts

$$
\alpha\left(I^{(m)}\right)>m \cdot \sqrt{s}
$$

for $s \geq 10$ general points in $\mathbb{P}^{2}$. Despite tremendous efforts this conjecture remains open for more than half a century, see [7] for a nice overview and recent progress.

Turning back to Problem 1.5 we note that the constant $N-1$ appearing there is the most optimistic one, since again star configurations can be used in order to show that the statement would fail with $N$ in place of $N-1$. On the other hand there is number of cases where the question asked in Problem 1.5 has positive answer.

Theorem 3.8 (Evidence for Problem 1.5) The containment in Problem 1.5 holds

(a) for arbitrary ideals in characteristic 2;

(b) for monomial ideals in arbitrary characteristic;

(c) for ideals of $d$-stars;

(d) for ideals of general points in $\mathbb{P}^{2}$ and $\mathbb{P}^{3}$.

Proof Claim (a) follows from a private communication from Huneke to Harbourne mentioned in [3, Example 8.4.4]. Claim (b) is an application of the Postulation Containment Criterion as explained in [3, Example 8.4.5]. Part (c) is again an application of Proposition 3.6 based on numerical data provided in [3, Lemma 8.4.7]. Finally claim (d) is proved for points in $\mathbb{P}^{2}$ in [18, Proposition 6.10] (it follows also from [5, Remark 4.3]) and for points in $\mathbb{P}^{3}$ in [11, Theorem 3].

The first counterexample to Problem 1.4 and hence also to Problem 1.5 was announced in [14]. We report on it in details in the subsequent section. Now we mention counterexamples to Problem 1.5 announced recently by Harbourne and Seceleanu in [19]. These are up to date the only counterexamples known in higher dimensional projective spaces. Such examples are available only in positive characteristic.

From now on let $\mathbb{K}$ be a field of odd characteristic $p$ and let $\mathbb{L}$ be its subfield of order $p$. 
Counterexample 3.9 Let $N=\frac{p+1}{2}$ and let $Z$ be the set of all but one $\mathbb{L}$-points in $\mathbb{P}^{N}(\mathbb{K})$. Then for the ideal $I=I(Z)$ there is

$$
I^{\left(\frac{p+3}{2}\right)} \nsubseteq I^{2}
$$

Proof The key observation is that whereas $\alpha\left(I^{2}\right)=p^{2}+1$, there is in the ideal $I^{\left(\frac{p+3}{2}\right)}$ a form of degree $p^{2}$, see [19, Theorem 3.9] for details.

The second counterexample allows more flexibility in the choice of the ordinary power.

Counterexample 3.10 We choose now the numbers $p$ and $N$ so that $p \equiv 1(\bmod N)$ and $p>(N-1)^{2}$. Let again $Z$ be the set of all but one $\mathbb{L}$-points in $\mathbb{P}^{N}(\mathbb{K})$. Then for $r=\frac{p-1}{N}+1$ there is

$$
I^{(p)} \nsubseteq I^{r}
$$

Proof See [19, Theorem 3.10].

We conclude this section recalling a homological containment criterion invented recently by Seceleanu, see [27, Theorem 3.1]. The criterion involves local homology. The underlying idea is quite simple, the containment $I^{(m)} \subset I^{r}$ induces a map between local cohomology modules $H_{M}^{0}\left(R / I^{m}\right) \rightarrow H_{M}^{0}\left(R / \mathcal{I}^{r}\right)$.

Theorem 3.11 (Seceleanu) Let $I \subset R$ be a homogeneous ideal. Consider the associated exact sequence

$$
0 \rightarrow I^{r} / I^{m} \rightarrow R / I^{m} \stackrel{\pi}{\rightarrow} R / I^{r} \rightarrow 0 .
$$

Then the following conditions are equivalent:

(i) there is the containment $I^{(m)} \subset I^{r}$,

(ii) the induced map $H_{M}^{0}(\pi): H_{M}^{0}\left(R / I^{m}\right) \rightarrow H_{M}^{0}\left(R / I^{r}\right)$ is the zero map.

There is also the dual version involving the Ext functor. In fact this dual approach together with the minimal free resolutions of the involved ideals open door to an effective application of Theorem 3.11. Even in the case of points in a plane this is a non-trivial operation. Seceleanu developed additional arguments [27, Theorem 3.3] in order to deal just with the case $\mathcal{I}^{(3)} \subset$ $\mathcal{I}^{2}$. Her method was successfully applied in order to verify the non-containment for the Klein configuration of points and for the series of Fermat examples, see Sect. 4.

\section{Specific statements related to Problem 1.4}

The first counterexample to Problem 1.4 was announced in 2013 by Dumnicki, TutajGasińska and the first author in [14]. Afterwards whole series of further counterexamples in all characteristics but 2 (see Theorem 3.8 (a)) have been found. Strangely enough all these counterexamples arise taking as the support of $I$ all (or almost all) intersection points of configurations of lines either defined by reflection groups or distinguished in some other way. Such configurations are extremal also from the combinatorial point of view, e.g. they exhibit an unusually high number of intersection points of high multiplicity. This is a surprising phenomenon not understood yet. 
Counterexample 4.1 (Dual Hesse configuration) Let $\varepsilon$ be a primitive root of 1 of order 3. We consider the saturated and radical ideal $I$ of the following set of 12 points in the complex projective plane $\mathbb{P}^{2}$ :

$$
\begin{aligned}
& P_{1}=(1: 0: 0), \quad P_{2}=(0: 1: 0), \quad P_{3}=(0: 0: 1), \\
& P_{4}=(1: 1: 1), \quad P_{5}=\left(1: \varepsilon: \varepsilon^{2}\right), \quad P_{6}=\left(1: \varepsilon^{2}: \varepsilon\right), \\
& P_{7}=(\varepsilon: 1: 1), \quad P_{8}=(1: \varepsilon: 1), \quad P_{9}=(1: 1: \varepsilon), \\
& P_{10}=\left(\varepsilon^{2}: 1: 1\right), P_{11}=\left(1: \varepsilon^{2}: 1\right), P_{12}=\left(1: 1: \varepsilon^{2}\right) \text {. }
\end{aligned}
$$

Then

$$
I^{(3)} \nsubseteq I^{2}
$$

Proof See [14, Theorem 2.2].

The points listed in Counterexample 4.1 are all intersection points of an arrangement of 9 lines given explicitly by equations

$$
\begin{aligned}
& L_{1}: x-y=0, \quad L_{2}: y-z=0, \quad L_{3}: z-x=0, \\
& L_{4}: x-\varepsilon y=0, \quad L_{5}: y-\varepsilon z=0, \quad L_{6}: z-\varepsilon x=0, \\
& L_{7}: x-\varepsilon^{2} y=0, \quad L_{8}: y-\varepsilon^{2} z=0, \quad L_{9}: z-\varepsilon^{2} x=0 .
\end{aligned}
$$

The product of these 9 linear equations is an element in $I^{(3)}$ (since there are exactly 3 lines passing through any of the points $P_{1}, \ldots, P_{12}$ but this product is not contained in $I^{2}$. Up to projective change of coordinates there is just one configuration of this kind, see [1] for this and much more. It is also well known that such a configuration cannot exist in the real projective plane because it would violate the celebrated Sylvester-Gallai Theorem. Nevertheless a real counterexample has been discovered shortly after announcement of [14]. It is presented in [9]. Quite expectedly this counterexample is related to constructions of real line configurations with the maximal number of triple points.

Counterexample 4.2 (Böröczky configurations) It is convenient to identify the real plane $\mathbb{R}^{2}$ with the set of complex numbers $\mathbb{C}$ in the usual way. Let $n$ be an even and positive integer. Let $\xi=\exp (2 \pi i / n)$ be a primitive $n$-th root of unity and let $P_{i}=\xi^{i}$ for $i=0, \ldots, n-1$ be vertices of a regular $n$-gon. For $m, k \in\{0, \ldots, n-1\}$ we denote by $L_{m, k}$ the (real) line determined by the points $P_{m}$ and $P_{k}$ if $m \neq k$ and the tangent line to the unit circle at the point $P_{m}$ if $m=k$.

The configuration of lines we are interested in is defined as the union of $n$ lines

$$
\mathcal{L}_{n}=\left\{L_{i, \frac{n}{2}-2 i}, i=0, \ldots n-1\right\},
$$

where the indices are understood $\bmod n$. Let $Z_{n}$ be the set of all triple points in the configuration $\mathcal{L}_{n}$. Such a configuration, for $n=12$ is visualized in Fig. 1 . The 19 points in the set $Z_{12}$ are marked by dots.

Let $I$ be the saturated radical ideal defined by $Z_{12}$. Then

$$
I^{(3)} \nsubseteq I^{2} \text {. }
$$

Proof The main idea is again to show that the product

$$
L_{1} \cdot L_{2} \cdot \ldots \cdot L_{9}
$$

of linear forms defining the lines $L_{i}$ is an element in $I^{(3)}$ which is not contained in $I^{2}$. See [9, Theorem 3]. 


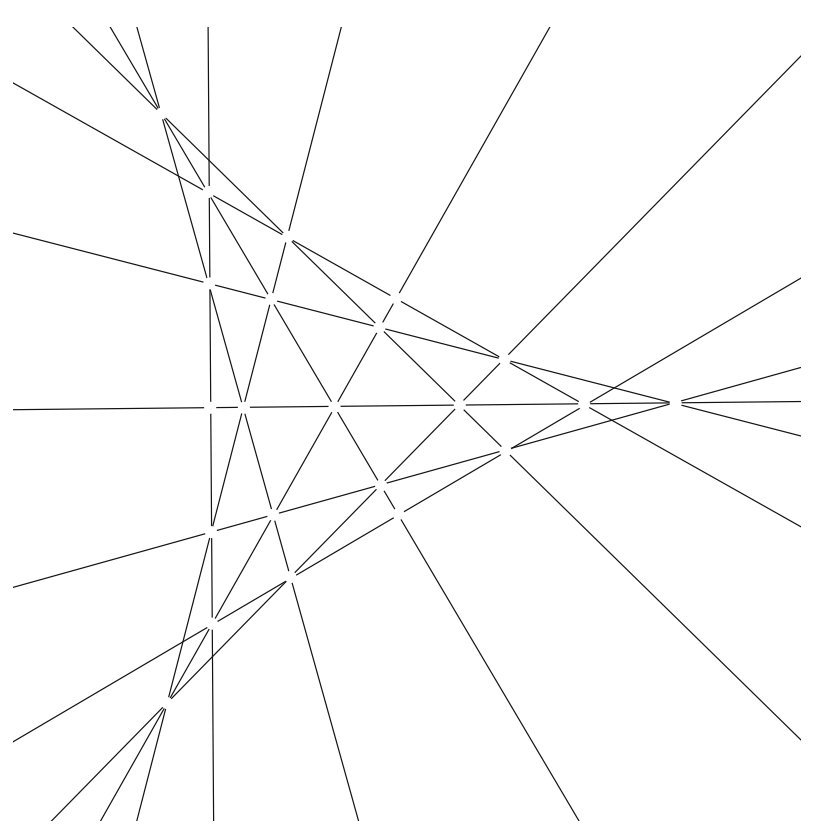

Fig. 1 Böröczky configuration of 12 lines

Contrary to the dual Hesse configuration, the configuration of 12 lines outlined above allows much more freedom. It has been noticed already in [12] that it can be perturbed into a configuration of lines in the rational projective plane, see the discussion on page 389 of [12]. The moduli space of all such configurations has been studied in more detail by LampaBaczyńska and the second author with the result that they are parameterized by a threedimensional rational variety, see [25, Theorem A]. It came as a surprise that the analogous configuration of 15 lines allows only 1 parameter moving along an explicitly determined elliptic curve. In that case there are no rational configurations possible, see [25, Theorem B].

Turning back to a more general setting, we observe first that Counterexample 4.1 is a special case of a whole series of configurations of lines leading to counterexamples to Problem 1.4. This series has been studied (with a different motivation) by Urzua in [30, Example II.6].

Counterexample 4.3 (Fermat configurations) Let $n \geq 3$ be an integer and let $\mathbb{K}$ be a field of characteristic different from 2 containing $n$ distinct roots of 1 . The polynomial

$$
F_{n}(x, y, z)=\left(x^{n}-y^{n}\right)\left(y^{n}-z^{n}\right)\left(z^{n}-x^{n}\right)
$$

splits completely into $3 n$ linear forms. They define lines which intersect in 3 points of multiplicity $n$ and $n^{2}$ points of multiplicity 3. If $\eta$ is a primitive root of 1 of order $n$, then the intersection points of these lines are $Q_{a, b}=\left(1: \eta^{a}: \eta^{b}\right)$ for $a, b=1 \ldots, n$ and the three coordinate points $P_{1}=(1: 0: 0), P_{2}=(0: 1: 0)$ and $P_{3}=(0: 0: 1)$. There are exactly $n$ lines meeting in a coordinate point and exactly 3 lines passing through every point $Q_{a, b}$. Taking $I$ as the radical ideal of the union of all points $Q_{a, b}$ and $P_{i}$, it is easy to see that $F_{n} \in I^{(3)}$. It is also easy to identify $I$ explicitly as

$$
I=\left\langle x\left(y^{n}-z^{n}\right), y\left(z^{n}-x^{n}\right), z\left(x^{n}-y^{n}\right)\right\rangle .
$$


It requires however some effort to show that $F_{n}$ is not contained in $I^{2}$ so that we have

$$
I^{(3)} \nsubseteq I^{2} \text {. }
$$

Proof This is Proposition 2.1 in [19]. An alternative proof is presented in [27, Proposition 4.1].

Additional counterexamples are in a sense isolated and they come from reflection groups acting on $\mathbb{P}^{2}$.

Counterexample 4.4 (Klein configuration) The configuration of bitangents of a smooth quartic curve in $\mathbb{P}^{2}$ has been a classical object of study. In particular for the Klein curve given by the equation

$$
x y^{3}+y z^{3}+z x^{3}=0
$$

it is highly symmetric. This has been first discovered and studied by Klein in [23]. The Klein curve is the unique plane quartic curve with the group of automorphisms of the maximal size of 168 elements, see [21] for a modern treatment. This group is the $\mathrm{PSL}_{3}(2)$ and it contains 21 involutions whose fixed lines form a configuration of 21 lines whose all incidences are 21 points where the lines meet by 4 and 28 points incident each to 3 lines. This is the Klein configuration, one of few known configurations with no simple intersection points, i.e. points incident to two lines only. Taking $I$ to be the ideal of all 49 intersection points mentioned above, we have

$$
I^{(3)} \nsubseteq I^{2}
$$

Proof See [27, Theorem 4.4] for an even stronger statement.

The next configuration arises also from involutions in a reflection group of order 360 , see [31].

Counterexample 4.5 (Wiman configuration) The Wiman configuration consists of 45 lines which intersect 120 triple, 45 quadruple and 36 quintuple points. Taking as $I$ the ideal of all these intersection points we have

$$
I^{(3)} \nsubseteq I^{2}
$$

Proof This claim has been so far verified only by computer. We used Singular [10].

Remark 4.6 (A link to the bounded negativity conjecture) Interestingly the last two counterexamples have appeared recently also in a seemingly unrelated topic of bounding Harbourne constants on birational models of $\mathbb{P}^{2}$, see [2]. It would be desirable to understand if there are some closer links between the Bounded Negativity Conjecture and the Containment Problem.

Before we pass to counterexamples in finite characteristic we point out the following.

Remark 4.7 In characteristic 0 so far there are no counterexamples to Problem 1.5, apart of those for $r=2, m=3$ and $N=2$.

The situation is quite different for points in projective planes in finite characteristic. Shortly after Counterexample 4.1 has been found, Bocci, Cooper and Harbourne pointed out in [4, Remark 3.11] that there is a similar example in characteristic 3. Indeed the combinatorics of the two configurations is exactly the same. Nevertheless there are discrepancies between other algebraic invariant, e.g. the resurgence, see [12, Theorem 2.1 and Theorem 3.2]. 
Counterexample 4.8 (Lines in $\mathbb{P}^{2}\left(\mathbb{F}_{3}\right)$ ) Let $Z$ consist of all but one points in $\mathbb{P}^{2}\left(\mathbb{F}_{3}\right)$. Then there are 12 points in $Z$, in each of which exactly 3 out of 9 lines missing the removed point meet. Then the containment 3 fails for the ideal of $Z$.

Proof This is of course a special case of Counterexample 3.9.

The next counterexample is a finite characteristic version of Counterexample 4.4.

Counterexample 4.9 (Conic in $\mathbb{P}^{2}\left(\mathbb{F}_{7}\right)$ ) It is well known that the Klein quartic reduces mod 7 to a smooth (double) conic $C$ in $\mathbb{P}^{2}\left(F_{7}\right)$. The bitangents of the quartic correspond then to the secant lines of the conic. There are 28 of them. There are 8 more tangent lines to the conic and 21 lines which do not meet $C$. These 21 lines form again a configuration with 21 quadruple intersection points and 28 triple intersection points. Taking as $Z$ the set of all these intersection points the containment

$$
I^{(3)} \subset I^{2}
$$

fails for the ideal $I$ of $Z$.

\section{Alternative approach}

Problem 1.5 focuses on exponents on both sides of the containment

$$
I^{(m)} \subset I^{r} .
$$

It is however possible to sharpen the assertions of Theorem 1.3 by making the ideal on the right smaller in another way. This line of investigation was suggested in [18]. This approach was motivated by the following conjecture due to Eisenbud and Mazur.

Conjecture 5.1 (Eisenbud-Mazur) Let $P \subset \mathbb{C}\left[\left[x_{1}, \ldots, x_{N}\right]\right]$ be a prime ideal in the ring of formal power series. Then

$$
P^{(2)} \subset M \cdot P,
$$

where $M=\left\langle x_{1}, \ldots, x_{N}\right\rangle$.

Thus the key idea in this path of investigation, paralleling Problem 1.1 is to study the following question, see [18, Question 1.3].

Problem 5.2 (Harbourne, Huneke) Decide for which $m, r$ and $j$ there is the containment

$$
I^{(m)} \subset M^{j} I^{r} .
$$

For ideals defining fat points, Harbourne and Huneke formulated the following bold statement, see [18, Conjecture 2.1].

Conjecture 5.3 (Harbourne-Huneke) Let $I \subset \mathbb{K}\left[x_{0}, \ldots, x_{N}\right]$ be a fat points ideal and let $r$ be a positive integer. Then the containment

$$
I^{(m)} \subset M^{r(N-1)} I^{r}
$$

holds for all $m \geq r N$.

를 Springer 
Positive results towards this Conjecture have been obtained in [18, Proposition 3.3] for fat points ideals in $\mathbb{P}^{2}$ arising as symbolic powers of radical ideals generated in a single degree. Particular examples of such ideals are star configurations of points and general points whose number is a binomial coefficient.

Another classes of ideals supporting Conjecture 5.3 have been studied in [13, Theorem B].

To the best of our knowledge there is no counterexample to Conjecture 5.3 known at present.

\section{A list of potential problems to think about}

The article [4] contains a long list of conjectures, including Problem 1.5 and Conjecture 5.3 discussed in the present note. Some of these conjectures require adjustments since the counterexamples presented here show their failure as well. These conjectures are also redundant in the sense that under some additional conditions some of them are stronger than the others. The picture presented in [4] is a little bit messy. For this reason, rather than repeating the conjectures and attempting to account on their current state, we have decided to present here a short list of open problems which are probably less challenging than Conjecture 5.3 on the one hand but also more accessible on the other hand.

It would be desirable to improve the Postulation Containment Criterion. The left hand side in the condition in Proposition 3.6 is often used in order to bound the regularity of the power $I^{r}$. It is natural to wonder if one can use this regularity directly.

Question 6.1 Can one replace $r \operatorname{reg}(I)$ by $\operatorname{reg}\left(I^{r}\right)$ in Proposition 3.6?

The next question presents a similar approach based on another interesting invariant studied recently. Cutkosky, Herzog and Trung showed that the regularity of ordinary powers of a homogeneous ideal is eventually linear. An analogous question for asymptotic regularity of symbolic powers of ideals is far more involved. In the case relevant here, i.e. in the case of ideals with zero-dimensional support it has been studied recently by Cutkosky and Kurano in [8] in a slightly more general setting of weighted projective spaces.

Definition 6.2 (Symbolic asymptotic regularity) Let $I$ be a homogeneous ideal. The real number

$$
\operatorname{areg}(I)=\lim _{m \rightarrow \infty} \frac{\operatorname{reg}\left(I^{(m)}\right)}{m}
$$

is the symbolic asymptotic regularity of $I$.

Cutkosky and Kurano showed that the limes in Definition 6.2 actually exists and it is equal to the so called $s$-invariant of $I$, which in turn is the reciprocal of the multi-point Seshadri constant of the reduced subscheme defined by $I$.

Question 6.3 Can one replace $\operatorname{reg}(I)$ by $\operatorname{areg}(I)$ in Proposition 3.6?

In the view of counterexamples presented in this note, an obvious attempt in order to save as much as possible of Problem 1.5 is the following statement.

Question 6.4 Let $I$ be a homogeneous radical ideal of a finite set of points in $\mathbb{P}^{N}$ with $N \geq 3$. Let $r$ be a positive integer. Does then the containment

$$
I^{(m)} \subset I^{r}
$$

hold for all $m \geq N r-1$ ? 
Thus a more specific question replacing in a sense Problem 1.4 is the following.

Question 6.5 Let $I$ be the radical ideal of a finite set of points in $\mathbb{P}^{3}$. Is then always

$$
I^{(5)} \subset I^{2} ?
$$

The next problem is motivated by Conjecture 5.1 even though it would not be a simple consequence of the Conjecture, see [4, Question 2.5].

Question 6.6 Let $I$ be an arbitrary homogeneous ideal in $R$. Is then

$$
I^{(j+1)} \subset M I^{(j)}
$$

for all $j \geq 1$ ?

Acknowledgements Research presented in this note has been started during the workshop "Recent advances in Linear series and Newton-Okounkov bodies" held in Universitá degli Studi di Padova in February 2015. We would like to thank the organizers for invitation to participate in the workshop and for excellent working conditions and stimulating atmosphere they have created. Finally we would like to thank the referee for helpful comments. We were partially supported by National Science Centre, Poland, Grant 2014/15/B/ST1/02197.

Open Access This article is distributed under the terms of the Creative Commons Attribution 4.0 International License (http://creativecommons.org/licenses/by/4.0/), which permits unrestricted use, distribution, and reproduction in any medium, provided you give appropriate credit to the original author(s) and the source, provide a link to the Creative Commons license, and indicate if changes were made.

\section{References}

1. Artebani, M., Dolgachev, I.: The Hesse pencil of plane cubic curves. L'Enseignement Mathématique. Revue Internationale. 2e Série 55, 235-273 (2009)

2. Bauer, Th, Di Rocco, S., Harbourne, B., Huizenga, J., Lundman, A., Pokora, P., Szemberg, T.: Bounded negativity and arrangements of lines. Int. Math. Res. Notices 2015, 9456-9471 (2015)

3. Bauer, Th., Di Rocco, S., Harbourne, B., Kapustka, M., Knutsen, A.L., Syzdek, W. and Szemberg, T.: A primer on Seshadri constants, pp. 33-70, In: Bates, D.J., Besana, G.-M., Di Rocco, S., Wampler, C.W. (eds.) Interactions of Classical and Numerical Algebraic Geometry, Proceedings of a Conference in Honor of A. J. Sommese, held at Notre Dame, May 22-24, 2008. Contemporary Mathematics, vol. 496 (2009)

4. Bocci, C., Cooper, S., Harbourne, B.: Containment results for various configurations of points in $\mathbb{P}^{N}$. J. Pure Appl. Algebra 218, 65-75 (2014)

5. Bocci, C., Harbourne, B.: Comparing powers and symbolic powers of ideals. J. Algebr. Geom. 19, 399-417 (2010)

6. Bocci, C., Harbourne, B.: The resurgence of ideals of points and the containment problem. Proc. Am. Math. Soc. 138, 1175-1190 (2010)

7. Ciliberto, C., Harbourne, B., Miranda, R., Roé, J.: Variations of Nagata's conjecture. In: A Celebration of Algebraic Geometry, pp. 185-203, Clay Math. Proc., vol. 18. Amer. Math. Soc., Providence (2013)

8. Cutkosky, S.D., Kurano, K.: Asymptotic regularity of powers of ideals of points in a weighted projective plane. Kyoto J. Math. 51, 25-45 (2011)

9. Czapliński, A., Główka, A., Malara, G., Lampa-Baczynska, M., Łuszcz-Świdecka, P., Pokora, P., Szpond, J.: A counterexample to the containment $I^{(3)} \subset I^{2}$ over the reals. Adv. Geom. 16 (2016), doi:10.1515/ advgeom-2015-0036

10. Decker, W., Greuel, G.-M.; Pfister, G., Schönemann, H.: Singular 4-0-2-a computer algebra system for polynomial computations. http://www.singular.uni-kl.de (2015)

11. Dumnicki, M.: Containments of symbolic powers of ideals of generic points in $\mathbb{P}^{3}$. Proc. Am. Math. Soc. 143, 513-530 (2015)

12. Dumnicki, M., Harbourne, B., Nagel, U., Seceleanu, A., Szemberg, T., Tutaj-Gasińska, H.: Resurgences for ideals of special point configurations in $\mathbb{P}^{N}$ coming from hyperplane arrangements. J. Algebra 443, 383-394 (2015)

13. Dumnicki, M., Szemberg, T., Tutaj-Gasińska, H.: A vanishing theorem and symbolic powers of planar point ideals. LMS J. Comput. Math. 16, 373-387 (2013) 
14. Dumnicki, M., Szemberg, T., Tutaj-Gasińska, H.: Counterexamples to the $I^{(3)} \subset I^{2}$ containment. J. Algebra 393, 24-29 (2013)

15. Ein, L., Lazarsfeld, R., Smith, K.: Uniform bounds and symbolic powers on smooth varieties. Invent. Math. 144, 241-252 (2001)

16. Eisenbud, D.: Commutative algebra. With a view toward algebraic geometry. Graduate Texts in Mathematics, vol. 150. Springer, New York (1995)

17. Geramita, A.V., Harbourne, B., Migliore, J.: Star configurations in $\mathbb{P}^{n}$. J. Algebra 376, 279-299 (2013)

18. Harbourne, B., Huneke, C.: Are symbolic powers highly evolved? J. Ramanujan Math. Soc. 28, 311-330 (2013)

19. Harbourne, B., Seceleanu, A.: Containment counterexamples for ideals of various configurations of points in $\mathbb{P}^{N}$. J. Pure Appl. Algebra 219, 1062-1072 (2015)

20. Hochster, M., Huneke, C.: Comparison of symbolic and ordinary powers of ideals. Invent. Math. 147, 349-369 (2002)

21. Jeurissen, R.H., van Os, C.H., Steenbrink, J.H.M.: The configuration of bitangents of the Klein curve. Discrete Math. 132, 83-96 (1994)

22. Keane, R.W., Küronya, A., McMahon, E.: An elementary approach to containment relations between symbolic and ordinary powers of certain monomial ideals. arXiv:1512.07092

23. Klein, F.: Über die Transformation siebenter Ordnung der elliptischen Functionen. Math. Ann. 14, 428471 (1879)

24. Lampa-Baczyńska, M., Malara, G.: On the containment hierarchy for simplicial ideals. J. Pure Appl. Algebra 219, 5402-5412 (2015)

25. Lampa-Baczyńska, M., Szpond, J.: From Pappus Theorem to parameter spaces of some extremal line point configurations and applications. Geom. Dedicata (to appear). arXiv:1509.03883

26. Nagel, U., Seceleanu, A.: Ordinary and symbolic Rees algebras for ideals of Fermat point configurations. J. Algebra 468, 80-102 (2016)

27. Seceleanu, A.: A homological criterion for the containment between symbolic and ordinary powers of some ideals of points in $\mathbb{P}^{2}$. J. Pure Appl. Algebra 219, 4857-4871 (2015)

28. Sidman, J., Sullivant, S.: Prolongations and computational algebra. Can. J. Math. 61, 930-949 (2009)

29. Swanson, I.: Linear equivalence of topologies. Math. Z. 234, 755-775 (2000)

30. Urzúa, G.A.: Arrangements of curves and algebraic surfaces. Thesis (Ph.D.) University of Michigan. ISBN: 978-0549-82049-9 (2008)

31. Wiman, A.: Über eine einfache Gruppe von 360 ebenen Collineationen. Math. Ann. 47, 531-556 (1896) 